Check for updates

Cite this: RSC Adv., 2019, 9, 33823

Received 3rd August 2019

Accepted 2nd October 2019

DOI: $10.1039 / c 9 r a 05180 e$

rsc.li/rsc-advances

\section{Long non-coding RNA DLX6-AS1/miR-141-3p axis regulates osteosarcoma proliferation, migration and invasion through regulating Rab10}

\begin{abstract}
Qiaoge Guo, ${ }^{a}$ Hui Sun, ${ }^{b}$ Kunpeng Zheng, ${ }^{b}$ Shaojie Yin ${ }^{c}$ and Junjie Niu (D)*d
Long non-coding RNA (IncRNAs) DLX6-AS1 plays significant roles in various types of malignant tumors, including osteosarcoma (OS), the most prevalent primary malignant bone tumor. However, the role and mechanism of DLX6-AS1 have not been fully illuminated in OS. Here, we aimed to find a novel mechanism for DLX6-AS1 in regulating the development of OS through sponging microRNA (miRNA). According to the luciferase reporter assay, RNA immunoprecipitation and RNA pull-down assay, miRNA (miR)-141-3p can physically interact with DLX6-AS1 and Rab10. The expressions of DLX6-AS1 and Rab10 were upregulated and miR-141-3p was downregulated in OS tissues and cells (MG-63 and U2OS), as described by RT-qPCR and western blotting. Moreover, there was a negative correlation between the expression of miR-141-3p and either DLX6-AS1 or Rab10, and a positive correlation between DLX6-AS1 and Rab10. Functionally, cell proliferation, migration and invasion were evaluated by utilizing the MTT assay and transwell assays. As a result, DLX6-AS1 knockdown suppressed OS cell proliferation, migration and invasion in MG-63 and U2OS cells, which was abolished by the downregulation of miR-141-3p. Similarly, the upregulation of Rab10 not only promoted OS cell progression in vitro, but also blocked the inhibitory effect of miR-141-3p overexpression in OS cells. Notably, DLX6-AS1 knockdown could, in turn, reverse the promoting effect of Rab10 on OS cell progression. Xenograft experiments depicted that DLX6-AS1 knockdown restrained the tumor growth of MG-63 cells in vivo. In conclusion, the knockdown of DLX6-AS1 might suppress OS progression via sponging miR-141-3p and downregulating Rab10, suggesting a novel DLX6-AS1/miR-141-3p/Rab10 pathway in OS progression.
\end{abstract}

\section{Introduction}

Osteosarcoma (OS) is the most prevalent primary malignant bone tumor in both adults and children. ${ }^{1}$ OS arises from primitive mesenchymal bone-forming cells, and it is characterized by early metastasis through the blood to other tissues, particularly the lungs., ${ }^{2,3}$ The current therapy of OS has been advancing, and radical surgery combined with neoadjuvant or adjuvant chemotherapy has been the most effective method. ${ }^{4}$ However, the 5 year overall survival rate of OS remains gloomy. ${ }^{5}$ Moreover, distant metastases and local recurrence are the major unfavorable factors for its prognosis, and the clinical prognosis of these metastatic or recurrent patients is extremely poor. ${ }^{6,7}$

${ }^{a}$ Department of CT \& MRI Imaging Diagnosis, Zhengzhou Orthopedic Hospital, Zhengzhou, Henan, China

${ }^{b}$ Department of Radiology, Zhengzhou Orthopedic Hospital, Zhengzhou, Henan, China ${ }^{c}$ Department of Radiology, Zhengzhou Second Hospital, Zhengzhou, Henan, China

${ }^{d}$ Department of Orthopedic, Shenzhen Pingle Orthopedic Hospital (Shenzhen Pingshan District Hospital of Traditional Chinese Medicine), No. 40, Jintang Street, Luohu District, Shenzhen, 518122, Guangdong, China. E-mail: uvfasy@163.com; Tel: +8615737177157
Thus, it is imperative and urgent to further explore new molecular mechanisms and therapeutic targets for OS.

Recent studies in human transcriptome analysis indicate that only $1-2 \%$ of transcripts of the human genome are protein-coding genes and the rest are non-coding RNAs, including long non-coding RNAs (lncRNAs) and small noncoding RNAs (microRNAs, miRNAs). ${ }^{\mathbf{8} 9}$ LncRNAs have more than 200 nucleotides, and miRNAs have approximately 22 nucleotides; both are known to play an important part in multiple biological processes, such as proliferation, migration, and invasion. ${ }^{\mathbf{1 0}}$ Acting as a tumor suppressor or oncogene, the dysregulation of IncRNAs and miRNAs is closely involved in the cellular functions and transformation activity in OS. ${ }^{11,12}$ Moreover, drug-associated lncRNAs and miRNAs have been discovered in OS. ${ }^{13}$ Therefore, emerging evidence suggests that lncRNAs and miRNAs are biomarkers and therapeutic targets for OS..$^{11,14}$

LncRNA distal-less homeobox 6 antisense 1 (DLX6-AS1) is a member of the DLX gene family. DLX6-AS1 is positively correlated with tumorigenesis in different tumors including hepatocellular carcinoma, pancreatic cancer, non-small cell lung cancer, ${ }^{15-17}$ and OS. ${ }^{18,19}$ However, the role and mechanism of DLX6-AS1 are still unclear in OS. Moreover, the tumor- 
suppressive role of miR-141-3p, a member of the miR-200 family, has been reviewed in OS. ${ }^{20}$ Research indicates that there is a link between miR-141-3p expression and OS cell behaviors, for instance, proliferation, apoptosis, autophagy, metastasis, and invasion. ${ }^{21-23}$ Furthermore, the overexpression of miR-141-3p might be closely related to the classification and size of OS. ${ }^{24}$ It is well-documented that lncRNAs, serving as a competitive endogenous RNA (ceRNA) sponge, target miRNAs to produce biological effects. Thus, we set out to determine whether there is a regulatory relationship between DLX6-AS1 and miR-141-3p in OS.

In this study, we examined the expression of DLX6-AS1 and miR-141-3p in OS tissues and cell lines. Moreover, functional experiments were performed to measure cell proliferation, migration and invasion in vitro, as well as tumor growth in vivo. We confirmed the regulatory pathway underlying DLX6-AS1 in OS progression through serving as a molecular "sponge" for miR-141-3p.

\section{Materials and methods}

\subsection{Acquisition of OS tissues}

With the approval of the Research Ethics Department of CT \& MRI Imaging Diagnosis, Zhengzhou Orthopedic Hospital and university and the written informed consents from 25 patients with primary OS, 25 paired femoral OS tissues and adjacent non-tumorous tissues were simultaneously obtained during operations from 2013 to 2017. All tissue samples were immediately stored in liquid nitrogen. The OS patients enrolled were pathologically confirmed and had not undergone any antitumor treatments, such as chemotherapy, immunotherapy, radiotherapy or radical surgery. This study is in compliance with the Declaration of Helsinki.

\subsection{Cells and cell culture}

Two human OS cell lines U2OS (HTB-96) and MG-63 (CRL-1427) were purchased from American Type Culture Collection (ATCC; Manassas, VA, USA) and one normal human osteoblast cell line NHost was obtained from the Chinese Cell Bank of the Chinese Academy of Sciences (Shanghai, China). These cells were cultured in Dulbecco's-modified Eagle's Medium (DMEM) (Gibco, Carlsbad, CA, USA) containing 10\% fetal bovine serum (FBS; Gibco) and $1 \%$ penicillin/streptomycin at $37{ }^{\circ} \mathrm{C}$ in a humidified atmosphere with $5 \% \mathrm{CO}_{2}$.

\subsection{Cell transfection}

Oligonucleotides siRNA against DLX6-AS1/NC (si-DLX6-AS1/ NC), miR-141-3p mimic/NC and miR-141-3p inhibitor/NC (inmiR-141-3p/NC) were purchased from Ribobio (Guangzhou, China). Plasmids pcDNA3.1-DLX6-AS1 and pcDNA3.1-Rab10 were constructed. Cell transfection of these oligonucleotides and plasmids into U2OS and MG-63 cells was performed using Lipofectamine 2000 (Invitrogen, Shanghai, China) according to the manufacturer's instructions. After transfection for $48 \mathrm{~h}$, U2OS and MG-63 cells were collected for further experiments except for the MTT assay.

\subsection{Real-time quantitative PCR (qPCR)}

Total RNA from tissue samples and cultured cells was isolated using TRIzol reagent (Thermo, Waltham, MA, USA) following the protocol. The first strand of cDNA was synthesized with total RNA as a template using a reverse transcription kit (Abcam, Cambridge, UK), and amplification of cDNA was performed by SYBR Premix Ex Taq Master Mix (TaKaRa, Shiga, Japan). qPCR was conducted on an Applied biosystem 7500 real-time PCR system (Thermo), and the expression of DLX6-AS1, Rab10 mRNA and miR-141-3p was calculated according to the comparative threshold cycle value $\left(2^{-\Delta \Delta C_{t}}\right)$ method, as compared with GAPDH or U6 small nuclear RNA (U6, for miRNA). PCR primers were as follows: DLX6-AS1: forward, $5^{\prime}$ AGTTTCTCTCTAGATTGCCTT-3 ${ }^{\prime}$; reverse, 5'-ATTGACATGTTAGTGCCCTT-3' ${ }^{\prime 25}$ miR-141-3p: forward, $5^{\prime}$ GGGCATCTTCCAGTACAGT-3' ${ }^{\prime}$ reverse, $5^{\prime}$ CAGTGCGTGTCGTGGAGT-3'; ; $^{26}$ Rab10: forward, 5'-CAAGGGAGCATGGTATTAGGTTT-3'; reverse, 5' ${ }^{\prime}$-CTAACGTGAGGAACGCCTTTT-3' ${ }^{\prime 27}$ GAPDH: forward, $5^{\prime}$ AGAGGCAGGGATGATGTTCTG-3' ${ }^{\prime}$; reverse, $5^{\prime}$-GACTCATGACCACAGTCCATGC-3'; U6: forward, 5'-CTCGCTTCGGCAGCACA-3'; reverse, 5'-AACGCTTCACGAATTTGCGT- ${ }^{\prime}$. All operations were carried out at least 3 times.

\subsection{Western blotting}

Transfected U2OS and MG-63 cells were extracted with total protein with RIPA lysis buffer (Beyotime, Shanghai, China). Western blotting was performed according to standard procedures, and $\beta$-actin on the same membrane was used as a loading control. The primary antibodies were as follows: anti-Rab10 (Abcam, \#110517, $1: 500$ ) and anti- $\beta$-actin (Abcam, \#8224, 1 : 5000). The proteins were visualized using the ECL procedure, and ImageJ was used to analyze the gray intensity of the bands.

\subsection{MTT assay}

3-(4,5-Dimethylthiazole-2-yl)-2,5-biphenyl tetrazolium bromide (MTT; Sigma, St Louis, USA) was used to evaluate the cell proliferative ability. Cells were seeded in a 96-well plate at the density of $4 \times 10^{3}$ cells per well overnight, then transfected with the corresponding oligonucleotides and plasmids. After transfection, the cells were cultured in normal medium for $0,24,48$, and $72 \mathrm{~h}$. In total, $5 \mathrm{mg} \mathrm{mL} \mathrm{m}^{-1}$ of MTT was added to DMEM and the cells were incubated for $4 \mathrm{~h}$, followed by incubation with 150 $\mu \mathrm{L}$ of DMSO (Sigma). The spectrophotometric absorbance of each sample was measured at $450 \mathrm{~nm}$. The experiments were conducted at least 3 times.

\subsection{Transwell assay}

Cell invasion and migration were measured using a Transwell chamber ( $8 \mu \mathrm{m}$ pore size, Corning, NY, USA) coated with or without matrigel (Becton Dickinson, Franklin Lakes, USA). Transfected MG-63 and U2OS cells were re-suspended in $200 \mu \mathrm{L}$ serum-free medium and then transferred into the upper chambers. The medium containing $10 \%$ FBS was used as a chemo-attractant and loaded into the low chamber. Transwell 
systems were kept at $37{ }^{\circ} \mathrm{C}$ for $24 \mathrm{~h}$. The cells that invaded and migrated into the lower chambers were stained with crystal violet and quantitated under a microscope. Three independent experiments were carried out.

\subsection{Luciferase reporter assay}

The potential binding between miR-141-3p and DLX6-AS1 and Rab10 was confirmed by luciferase reporter assays. The human DLX6-AS1 wild type (DLX6-AS1-WT), Rab10 $3^{\prime}$ untranslated region ( $\left.3^{\prime} \mathrm{UTR}\right)$ wild type (Rab10-WT) and its corresponding mutant type were cloned into psi-CHECK-2 (Promega, Madison, WI, USA). MG-63 and U2OS cells were co-transfected with miR141-3p/NC mimic with either DLX6-AS1-WT/MUT or Rab10-WT/ MUT. All transfection procedures were performed by Lipofectamine 2000 (Invitrogen). After $48 \mathrm{~h}$ of transfection, the luciferase activity was measured using a dual-luciferase reporter system (Promega) on a SpectraMaxM5 instrument software (Molecular Devices, Sunnyvale, USA). All operations were repeated three times.

\subsection{RNA immunoprecipitation (RIP) and RNA pull-down assay}

The RNA immunoprecipitation (RIP) and RNA pull-down assay were performed with MG-63 and U2OS cell extract. The Magna RIP ${ }^{\mathrm{TM}}$ RNA-binding protein immunoprecipitation kit (MilliporeSigma, Billerica, MA, USA) was chosen to enrich DLX6-AS1 from the samples bound to the Ago2 antibody or IgG. The co-precipitated RNAs were detected by RT-qPCR. Biotinylated (Bio-) DLX6-AS1-WT/MUT (Ribobio) and Bio-NC were separately transfected into MG-63 and U2OS cells, and the cell lysates were collected after transfection for $48 \mathrm{~h}$, followed by incubation with M-280 streptavidin magnetic beads (Invitrogen). The bound RNAs were analyzed using RTqPCR.

\subsection{Xenograft mouse model}

The 8 week-old athymic $\mathrm{BALB} / \mathrm{c}$ mice were obtained from Shanghai SLAC Laboratory Animal Co. Ltd. The animal experiments were approved by The Institutional Department of CT\&MRI Imaging Diagnosis, Zhengzhou Orthopedic Hospital and were untaken in accordance with National Institutes of Health Guide for Care and Use of Laboratory Animals. MG-63 cells were stably transfected with lentiviral particles encoding shRNA against DLX6-AS1 (sh-DLX6-AS1; Neuron Biotech, Shanghai, China) or the negative control (sh-NC) using Polybrene reagent (Sigma). Equal numbers $\left(10^{6}\right)$ of transfected MG-63 cells per $0.2 \mathrm{~mL}$ were subcutaneously injected into the flanks of mice ( 5 mice per group). The tumors were measured with a caliper once every 7 days, and the mice were euthanatized on day 28. The tumor volume was calculated using the formula: $0.5 \times a b^{2}$ ( $a$ is the longest tumor axis and $b$ is the shortest tumor axis). The weight of the tumors was determined using an electronic balance. Immediately, the tumors were frozen at $-80{ }^{\circ} \mathrm{C}$ for further isolation of total RNA and protein.

\subsection{Statistical analyses}

Data given were the mean \pm standard error; two-group comparisons were performed using Student's $t$-test on SPSS 17.0 (SPSS Inc., Chicago, IL, USA), and one-way analysis of variance (ANOVA) was for more than two groups. $P<0.05$ was identified to be statistically significant. The relationship between the expression levels of miR-141-3p and DLX6-AS1 and Rab10 mRNA in 25 OS tissues was analyzed by Spearman's correlation analysis.

\section{Results}

3.1. Expression of DLX6-AS1 was upregulated and miR-141$3 p$ was downregulated in OS tissues and cells

To confirm whether the DLX6-AS1/miR-141-3p axis exists in regulating OS progression, we firstly investigated their expression in patients with OS. As shown in Fig. 1A, the patients with OS had damaged femurs compared with the normal control as evidenced by magnetic resonance imaging (MRI). The expressions of DLX6-AS1 and miR-141-3p in femoral OS tissues and adjacent non-tumorous tissues were detected using RT-qPCR. We found that DLX6-AS1 levels were higher overall (Fig. 1B), and miR-141-3p levels were lower (Fig. 1D) in femoral OS tissues. Moreover, the expression of DLX6-AS1 was upregulated (Fig. 1C) and miR-141-3p was downregulated (Fig. 1E) in human OS cell lines MG-63 and U2OS as compared to the normal human osteoblast cell line NHost. Besides, the Spearman correlation analysis demonstrated a negative linear correlation between the expression of DLX6-AS1 and miR-141-3p (Fig. 1F, $r$ $=0.8086, P<0.0001)$. These outcomes suggested a potential regulatory relationship between the expression of DLX6-AS1 and miR-141-3p in OS cells.

\subsection{DLX6-AS1 negatively regulated miR-141-3p by sponging}

According to the starBase website, miR-141-3p was retrieved and identified as a possible target gene of DLX6-AS1. The sequences of the putative binding site in the DLX6-AS1-3' UTR wild type were mutated as the complementary sequences as shown in Fig. 2A. To confirm this, a dual-luciferase reporter assay was performed using psi-CHECK-2 plasmid, and MG-63 and U2OS cells were transfected with the miR-141-3p/NC mimic. Fig. 2B and C showed that the miR-141-3p mimic significantly reduced the relative luciferase activity of DLX6-AS1 wild type; whereas, there was little influence on the luciferase activity of DLX6-AS1 mutant when transfected with the miR-141-3p mimic or miR-NC mimic. In addition, RIP and RNA pull-down assays were carried out to further verify this target binding. As a result, the relative expression of DLX6-AS1 in MG-63 and U2OS cells was abundantly enriched by the Ago2 antibody when transfected with the miR-141-3p mimic (Fig. 2D), and the relative expression of miR-141-3p was dramatically pulled down in MG-63 and U2OS cells after the overexpressed bio-DLX6AS1 versus bio-NC transfection (Fig. 2E and F). Whereas, bioDLX6-AS1-MUT failed to distinctively improve the miR-141-3p expression. Next, the regulatory relationship between DLX6-AS1 and miR-141-3p was determined. We observed that the miR141-3p expression level was decreased when transfected with 
A

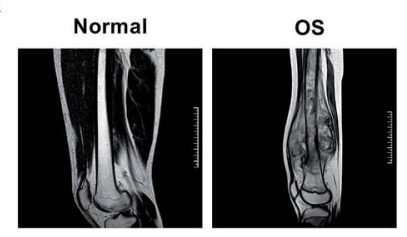

D

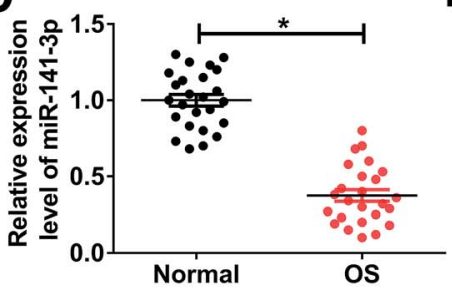

B

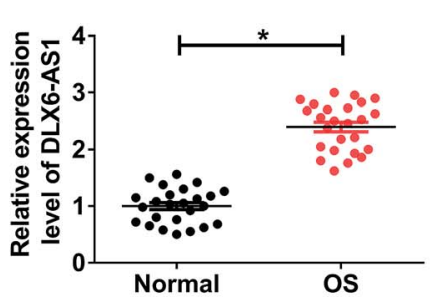

E

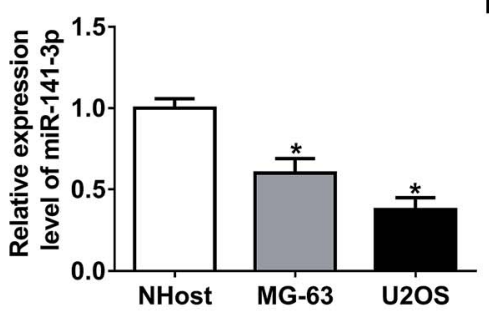

C

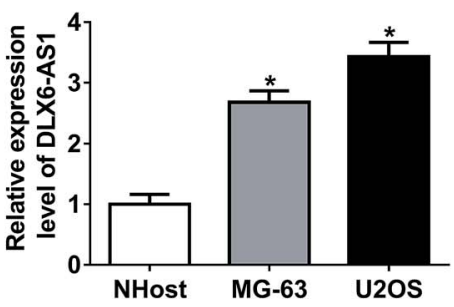

$\mathbf{F}$

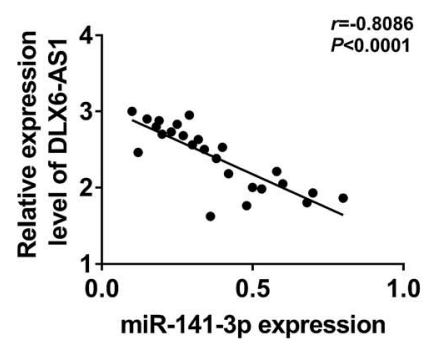

Fig. 1 Expression of IncRNA DLX6-AS1 (DLX6-AS1) and miRNA-141-3p (miR-141-3p) in osteosarcoma (OS). (A) Imaging features of femoral OS and the normal control using MRI. (B) DLX6-AS1 expression levels were detected by RT-qPCR in femoral OS tissues and adjacent non-tumorous (normal) tissues. Data are shown using $2^{-\Delta \Delta C_{T}}$ values. (C) DLX6-AS1 expression levels were determined in human OS cell lines MG-63 and U2OS, and the human osteoblast cell line NHost. ( $D$ and E) The expression of miR-141-3p was measured using RT-qPCR in femoral OS tissues and cell lines MG-63 and U2OS, as compared with normal tissue and NHost cells. (F) Analysis of the correlation between expressions of DLX6-AS1 and miR-141-3p in femoral OS tissues (Spearman correlation analysis). $* P<0.05$.

pcDNA-DLX6-AS1 and increased when transfected with si-DLX6AS1 (Fig. 2G). Taken together, our data proposes that DLX6-AS1 negatively regulated miR-141-3p in OS cells via target binding.

\subsection{Downregulation of miR-141-3p abolished the suppressive role of DLX6-AS1 knockdown in OS cells in vitro}

Subsequently, the role of the DLX6-AS1/miR-141-3p axis in OS cells in vitro was discovered. MG-63 and U2OS cells were transfected with si-DLX6-AS1/NC to examine DLX6-AS1 functions on cell proliferation, migration and invasion. As depicted in Fig. 3A and B, the cell proliferative ability of MG-63 and U2OS cells was obviously impaired by si-DLX6-AS1 transfection for 48$72 \mathrm{~h}$. Cell migration and invasion abilities of MG-63 and U2OS cells were analyzed with transwell assays. After the transfection of si-DLX6-AS1 for $48 \mathrm{~h}$, migratory cells and invasive cells were apparently descended (Fig. 3C and D). The data showed the suppressive role of DLX6-AS1 knockdown in OS cells in vitro, and then we wondered whether different expressions of miR141-3p influenced this effect. Therefore, rescue experiments were performed in MG-63 and U2OS cells when co-transfected with si-DLX6-AS1 and in-miR-141-3p or in-miR-NC. As expected, the inhibition of si-DLX6-AS1 in cell proliferation, migration and invasion was abolished in the presence of inmiR-141-3p (Fig. 3A-D). These results indicated that DLX6-AS1 knockdown exerted a tumor-suppressive role in OS cells through upregulating miR-141-3p.

\subsection{Rab10 was positively regulated by DLX6-AS1 via miR- 141-3p}

To further investigate the downstream targets of the DLX6-AS1/ miR-141-3p axis, we searched targetScan Human websites and observed a potential binding site for miR-141-3p in Rab10 3'UTR (Fig. 4A). The Rab10 3'UTR wild type containing the putative miR-141-3p target site were cloned into luciferase reporter vector psi-CHECK-2, as well as its corresponding mutant. The relative luciferase activity of the Rab10 wild type was significantly decreased in MG-63 and U2OS cells transfected with the miR-141-3p mimic (Fig. 4B and C); meanwhile, there was no difference in the Rab10 mutant groups. The expression of Rab10 mRNA was evaluated by RT-qPCR and was upregulated in human femoral OS tissues and cell lines MG-63 and U2OS as compared to the normal tissues and cell line NHost (Fig. 4D and E). Western blotting analysis indicated that Rab10 protein expression in MG-63 and U2OS cells was downregulated by both miR-141-3p mimic transfection and si-DLX6AS1 transfection (Fig. $4 \mathrm{~F}$ and $\mathrm{G}$ ). Furthermore, there was an inverse linear correlation (Fig. $4 \mathrm{H}, r=0.7792, P<0.0001$ ) between Rab10 mRNA expression and miR-141-3p expression, and a positive linear correlation (Fig. $4 \mathrm{I}, r=0.6628, P<0.0001$ ) between Rab10 mRNA expression and DLX6-AS1 expression in femoral OS tissues. Taken together, Rab10 was targeted by DLX6-AS1/miR-141-3p axis in OS cells.

\subsection{Upregulation of Rab10 blocked the suppressive role of miR-141-3p in OS cells in vitro}

The influence of Rab10 dysregulation on the role of DLX6-AS1/ miR-141-3p axis in OS cells was detected in vitro. MG-63 and U2OS cells were transfected with miR-141-3p/NC mimic to examine the miR-141-3p functions in cell proliferation, migration and invasion. As depicted in Fig. 5A and B, the cell proliferative capacity of MG-63 and U2OS cells was destroyed by miR-141-3p mimic transfection for 48-72 h. After 
A

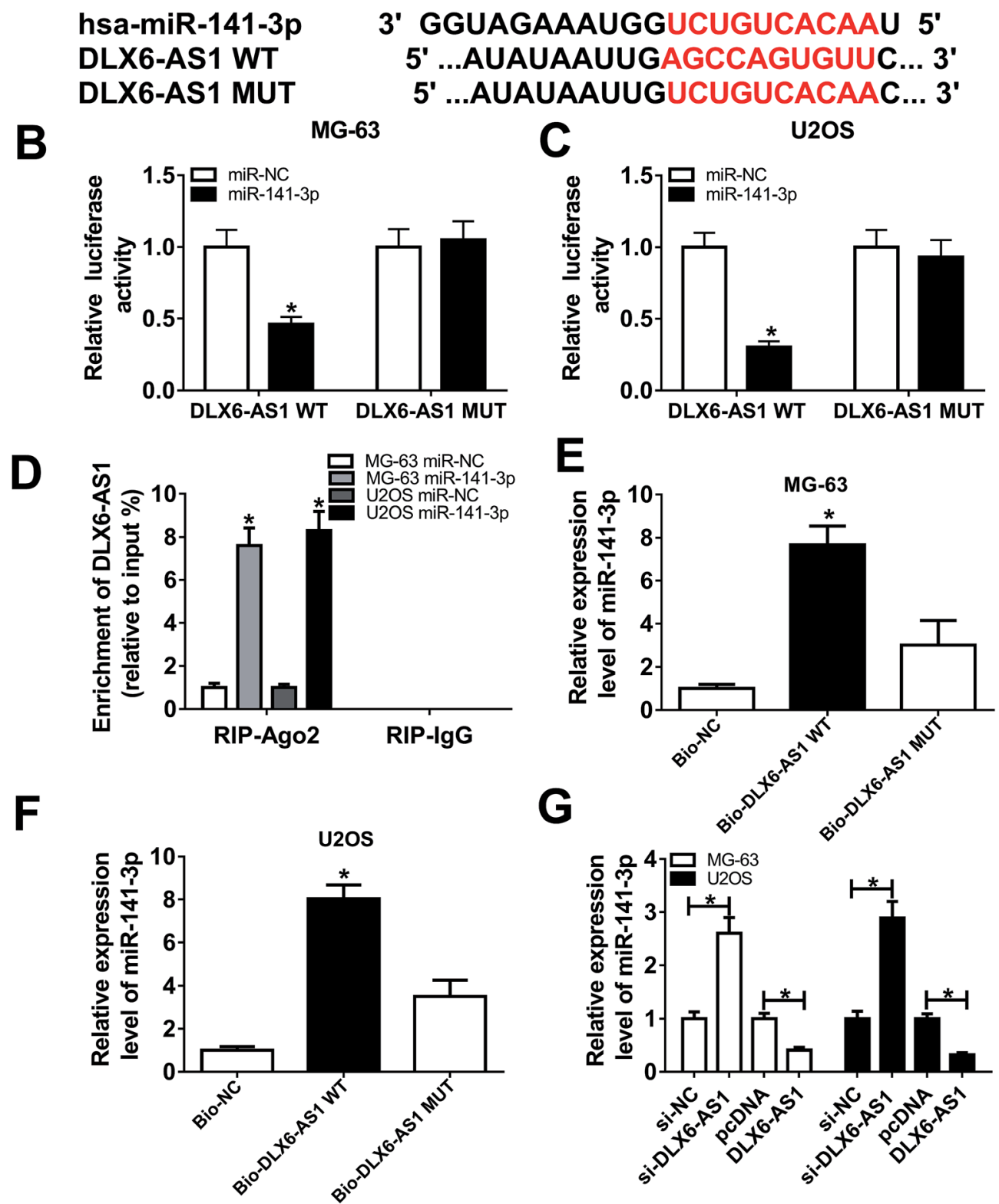

Fig. 2 DLX6-AS1 negatively regulated miR-141-3p by sponging. (A) Prediction of the potential binding site of miR-141-3p on DLX6-AS1 wild type (DLX6-AS1-WT) on starBase website. The putative targeting site was mutated as DLX6-AS1-MUT. (B and C) Relative luciferase activity of DLX6AS1-WT/MUT was examined in MG-63 and U2OS cells transfected with miR-141-3p/NC mimic (miR-141-3p/NC) using dual-luciferase reporter assay. (D) Relative enrichment of DLX6-AS1 in MG-63 and U2OS cells was measured using RNA immunoprecipitation assay when transfected with miR-141-3p or miR-NC. (E and F) Relative enrichment of miR-141-3p in MG-63 and U2OS cells was detected using the RNA pull-down assay after transfection with biotin-labelled NC (bio-NC), DLX6-AS1-WT (bio-DLX6-AS1) and DLX6-AS1-MUT (bio-DLX6-AS1-MUT). (G) MG-63 and U2OS cells were transfected with pcDNA-DLX6-AS1 (DLX6-AS1), siRNA against DLX6-AS1 (si-DLX6-AS1), and their negative controls. The expression level of miR-141-3p was measured after transfection. $* P<0.05$.

transfection of the miR-141-3p mimic for $48 \mathrm{~h}$, the abilities of cell migration and invasion were also inhibited (Fig. 5C and D). More importantly, the inhibition of miR-141-3p overexpression on cell viability, migration and invasion was significantly blocked with the ectopic expression of Rab10 (Fig. 5A-D). The data showed the suppressive role of miR-141$3 p$ overexpression in OS cells in vitro through the downregulating of its target Rab10.
3.6. Knockdown of DLX6-AS1 partially reversed the promoting role of Rab10 in OS cells in vitro

The biological activity of Rab10 overexpression in OS cells was identified, as well as the impact of DLX6-AS1 knockdown on its role. MG-63 and U2OS cells were transfected with pcDNA-Rab10 or pcDNA, and co-transfected with Rab10 and si-DLX6-AS1/NC. As depicted in Fig. 6A and B, the cell proliferative capacity of MG-63 and U2OS cells was promoted by pcDNA-Rab10 
A

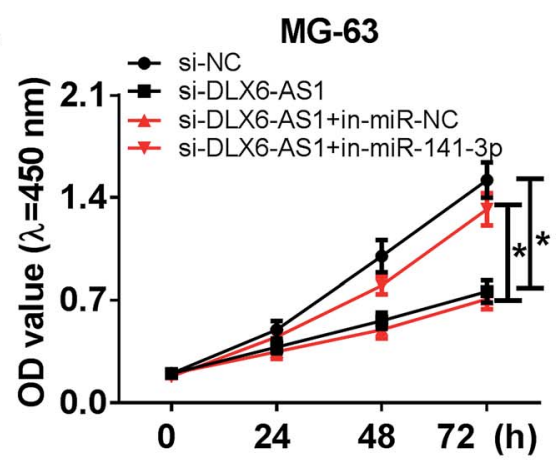

B

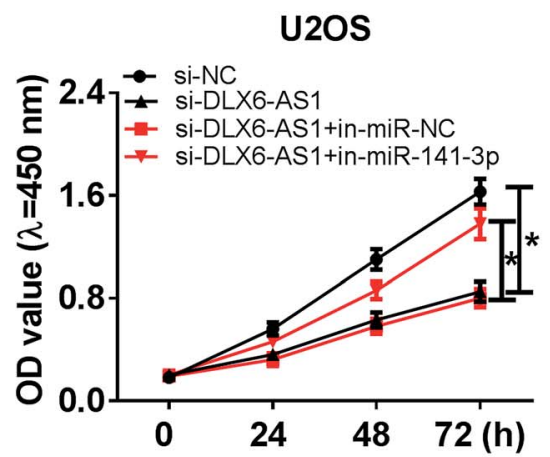

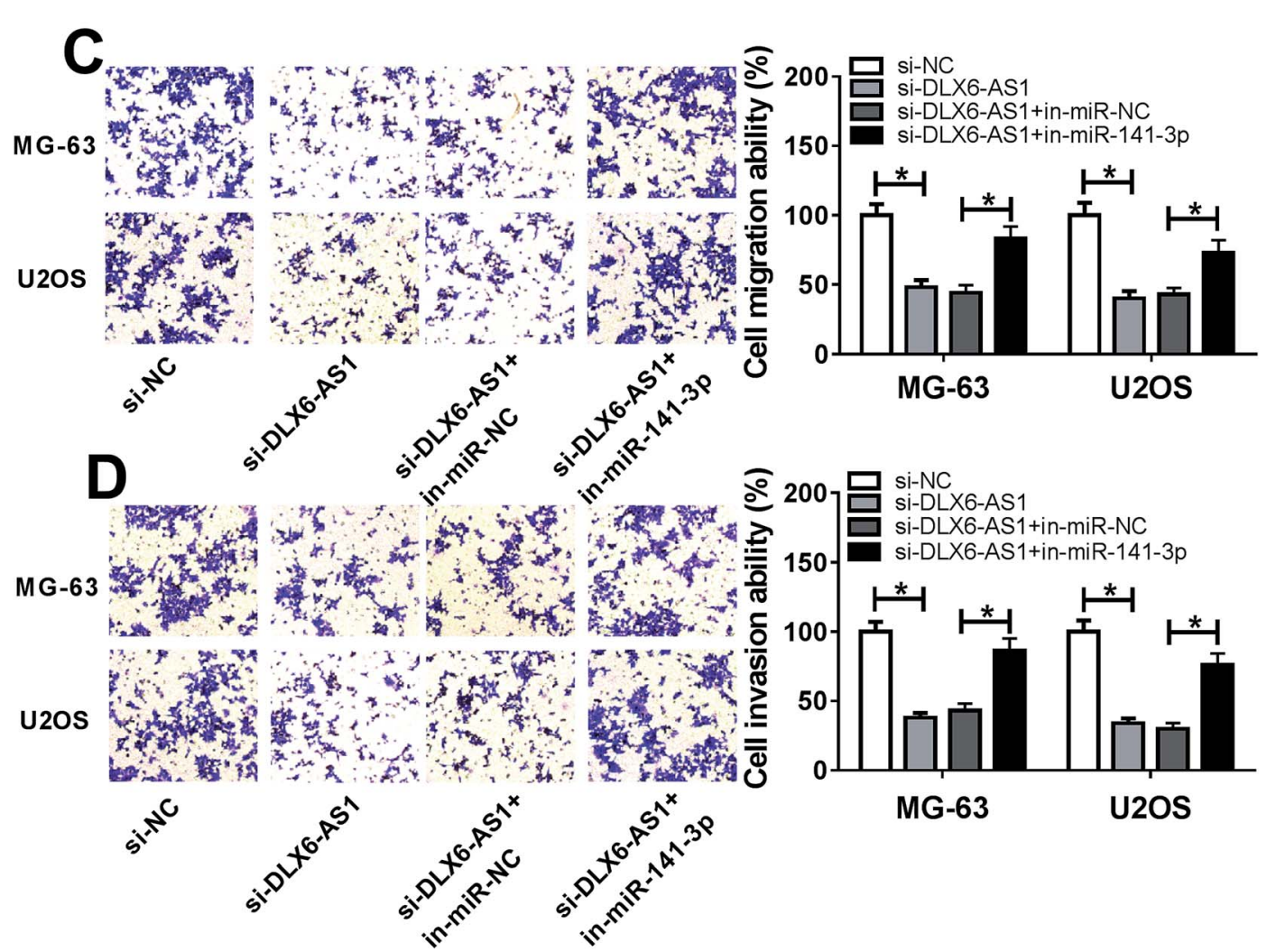

Fig. 3 Influence of miR-141-3p downregulation on the role of DLX6-AS1 knockdown in OS cells in vitro. MG-63 and U2OS cells were transfected with si-DLX6-AS1/NC or co-transfected with si-DLX6-AS1 and miR-141-3p/NC inhibitor (in-miR-141-3p/NC). (A and B) The cell proliferative ability of MG-63 and U2OS cells was evaluated by MTT assay after transfection for $0,24,48$ and $72 \mathrm{~h}$. (C and D) Cell migration and invasion abilities of MG-63 and U2OS cells were analyzed with transwell assays after transfection for $24 \mathrm{~h} . * P<0.05$.

transfection for $48-72 \mathrm{~h}$, and this was partially reversed in the presence of si-DLX6-AS1. Besides, the cell migration and invasion abilities were also facilitated with Rab10 overexpression, which was then countered by si-DLX6-AS1 transfection (Fig. 6C and D). These results indicated that Rab10, as a downstream target of DLX6-AS1/miR-141-3p axis, exhibited an oncogenic effect in OS cells in vitro, depending on DLX6-AS1 upregulation.

\subsection{Upregulation of DLX6-AS1 encountered the inhibition of miR-141-3p on Rab10 expression in OS cells in vitro}

We discovered that the dysregulation of DLX6-AS1 could, in turn, affect miR-141-3p effect. As shown in Fig. 7A and B, miR141-3p downregulated Rab10 mRNA and protein expression in
MG-63 and U2OS cells, while pcDNA-DLX6-AS1 transfection inhibited this downregulation. This outcome indicated that miR-141-3p downregulated Rab10 expression, depending on DLX6-AS1 deletion.

\subsection{Knockdown of DLX6-AS1 inhibited tumor growth of MG- 63 cells in vivo}

In order to verify the role of DLX6-AS1 in the tumorigenesis of OS, MG-63 cells were stably and lowly expressed with DLX6-AS1 and then injected into nude mice. As shown in Fig. 8A and B, the tumors were generated in both groups, and the tumors in the sh-DLX6-AS1 group were dramatically smaller and lighter than the sh-NC group after inoculation for 21-28 days. 
A

hsa-miR-141-3p Rab10 3'UTR-WT Rab10 3'UTR-MUT B

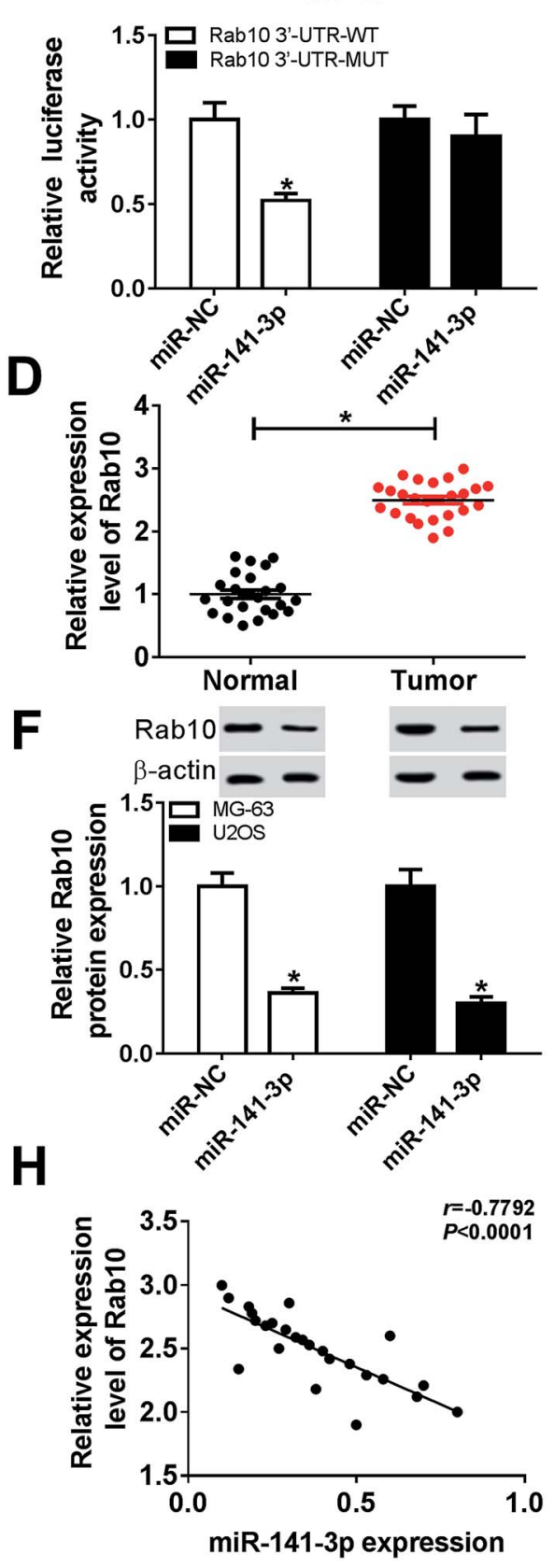

3' GguagaAAUgGuCUguCACAAU 5'

5'...UGAAGAACACUUUUUCAGUGUUA...3'

5'...UGAAGAACACUUUUUGUCACAAU....3'

C

U2OS
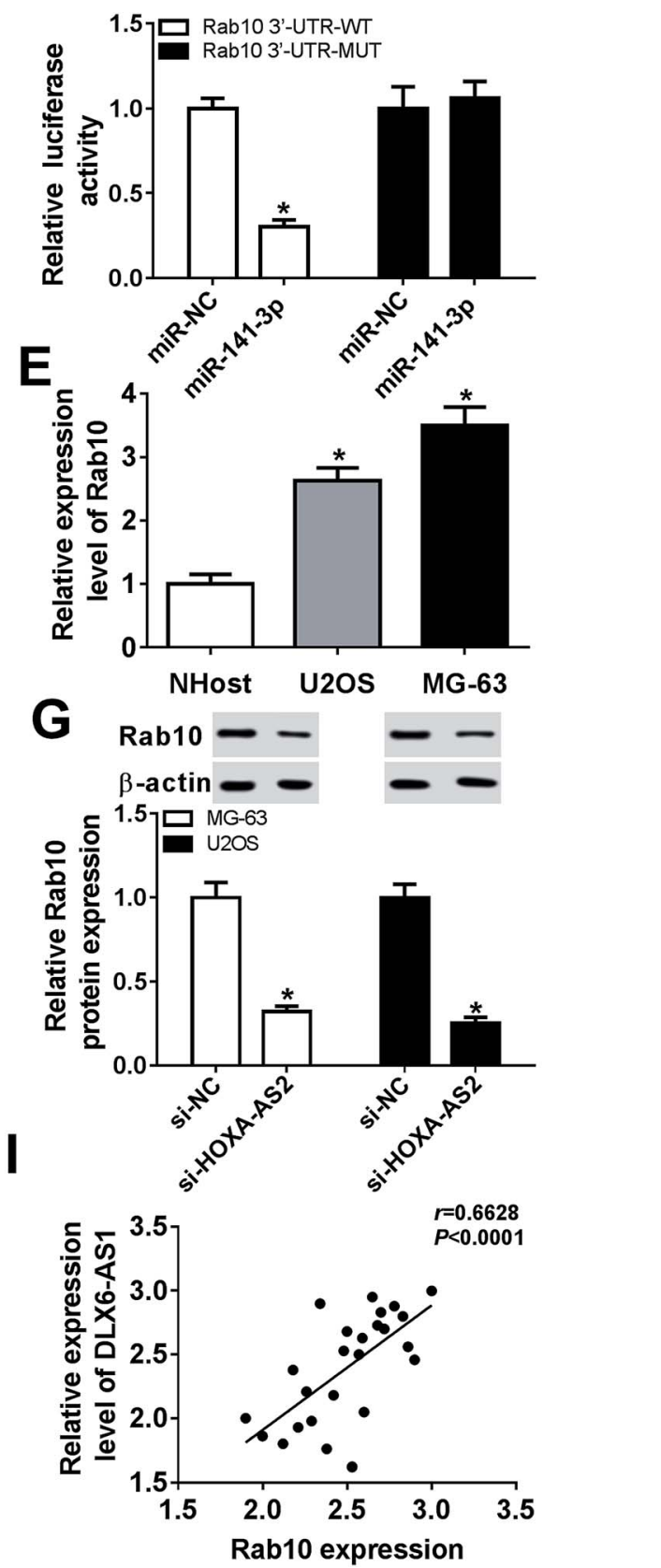

Fig. 4 Rab10 was a direct target of miR-141-3p and positively regulated by DLX6-AS1. (A) The potential binding site between miR-141-3p and Rab10 wild type (Rab10-WT) was predicted on the targetScan website. The putative targeting site was mutated as Rab10-MUT. (B and C) Relative luciferase activity of Rab10-WT/MUT was examined by dual-luciferase reporter assay in MG-63 and U2OS cells transfected with miR-141-3p/NC. (D and E) Expression of Rab10 mRNA was measured using RT-qPCR in femoral OS tissues and cell lines MG-63 and U2OS, compared with normal tissues and NHost cells. (F and G) Expression of Rab10 protein in MG-63 and U2OS cells was examined by western blotting with transfection of miR-141-3p/NC or si-DLX6-AS1/NC. (H and I) Analysis of the correlation between expressions of Rab10 mRNA and either DLX6-AS1 or miR-141$3 p$ in femoral OS tissues (Spearman correlation analysis, $r=0.8086, P<0.0001$ ). ${ }^{*} P<0.05$. 


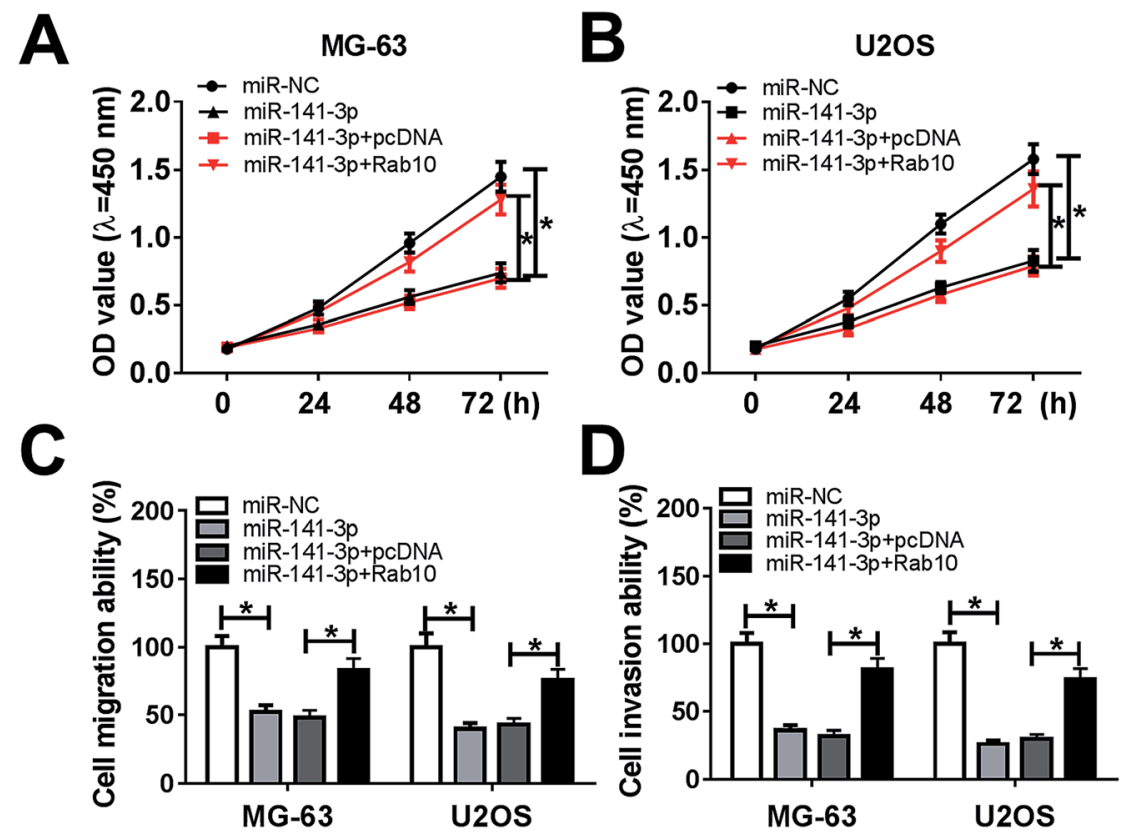

Fig. 5 Influence of Rab10 overexpression on the role of miR-141-3p in OS cells in vitro. MG-63 and U2OS cells were transfected with miR-141$3 p / N C$ or co-transfected with miR-141-3p and pcDNA (pcDNA) or pcDNA-Rab10 (Rab10). (A and B) Cell proliferative ability of MG-63 and U2OS cells was evaluated by MTT assay after transfection for 0, 24, 48 and 72 h. (C and D) Cell migration and invasion abilities of MG-63 and U2OS cells were analyzed with transwell assays after transfection for 48 h. $* P<0.05$.

Moreover, the expression of miR-141-3p was higher (Fig. 8D), and DLX6-AS1 and Rab10 were lower in xenograft tumors (Fig. 8C and E). These outcomes showed that the DLX6-AS1 knockdown inhibited the xenograft tumor growth, suggesting an association between DLX6-AS1 expression and the in vivo proliferative capacity of MG-63 cells.

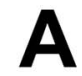

MG-63

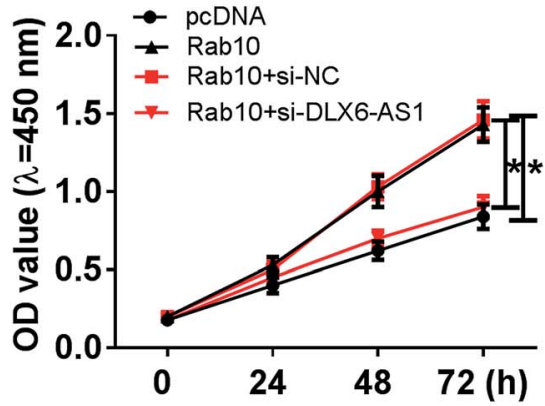

C

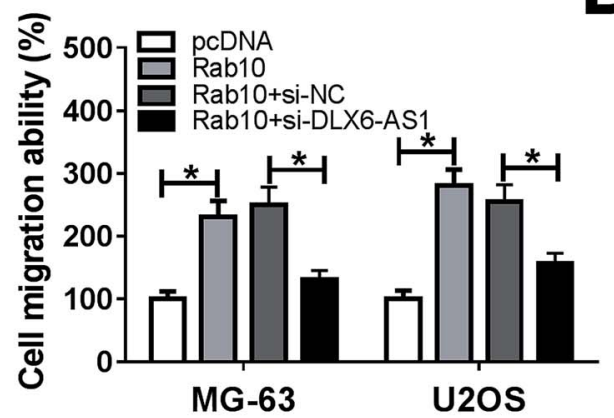

B U2OS
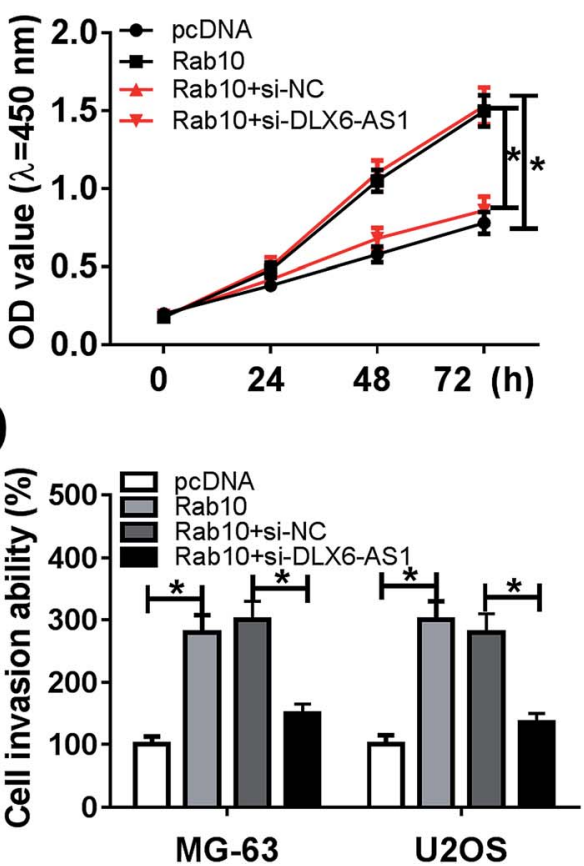

Fig. 6 Impact of DLX6-AS1 knockdown on the role of Rab10 in OS cells in vitro. MG-63 and U2OS cells were transfected with pcDNA and Rab10 or co-transfected with Rab10 and si-DLX6-AS1/NC. (A and B) Cell proliferative ability of MG-63 and U2OS cells was evaluated by MTT assay after transfection for $0,24,48$ and $72 \mathrm{~h}$. (C and D) Cell migration and invasion abilities of MG-63 and U2OS cells were analyzed with transwell assays after transfection for $48 \mathrm{~h} . * P<0.05$. 

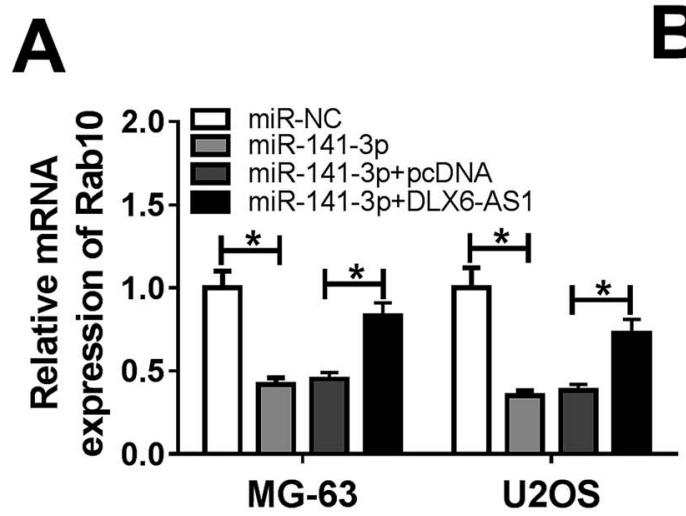

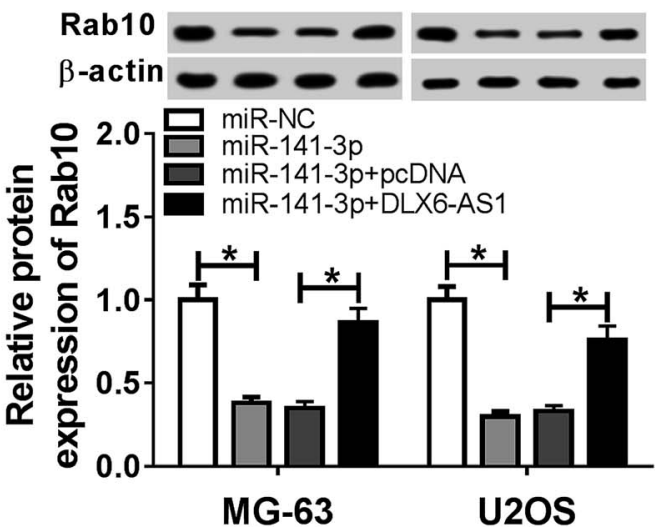

Fig. 7 Overexpression of DLX6-AS1 rescued miR-141-3p-mediated downregulation of Rab10. MG-63 and U2OS cells were transfected with miR-141-3p/NC or co-transfected with miR-141-3p and DLX6-AS1 or pcDNA. (A and B) Expressions of Rab10 mRNA and protein were detected after transfection for $48 \mathrm{~h}$ using RT-qPCR and western blotting, respectively. $* P<0.05$.

\section{Discussion}

DLX6-AS1 acts as a tumor suppressor in human malignant tumors, including OS. For example, downregulated DLX6-AS1 may inhibit the stem cell properties of liver cancer stem cells (LCSCs) as demonstrated by their inhibited spheroid formation, colony formation, proliferation, and tumor formation abilities. ${ }^{15}$ The knockdown of DLX6-AS1 suppressed cell growth in pancreatic cancer and NSCLC ${ }^{\mathbf{1 6 , 1 7}}$ is accompanied by decreased migration and invasion, and increased apoptosis in vitro. In this study, we observed high expression of DLX6-AS1 in femoral OS tissues and cells (MG-63 and U2OS), and its downregulation could impair proliferation, migration and invasion in OS cells in vitro, as well as tumor growth in vivo through sponging miR-141$3 \mathrm{p}$ and downregulating Rab10 expression. The expression of miR-141-3p was downregulated and Rab10 was upregulated in OS tissues and cells. Moreover, there was a linear correlation among the expressions of DLX6-AS1, miR-141-3p and Rab10.

DLX6-AS1 serves as ceRNA to exert biological functions and the DLX6-AS1/miRNAs/mRNAs network is an important molecular mechanism in tumors. Very recently, the effects of DLX6-AS1 in OS were investigated. Zhang et al. ${ }^{19}$ declared that

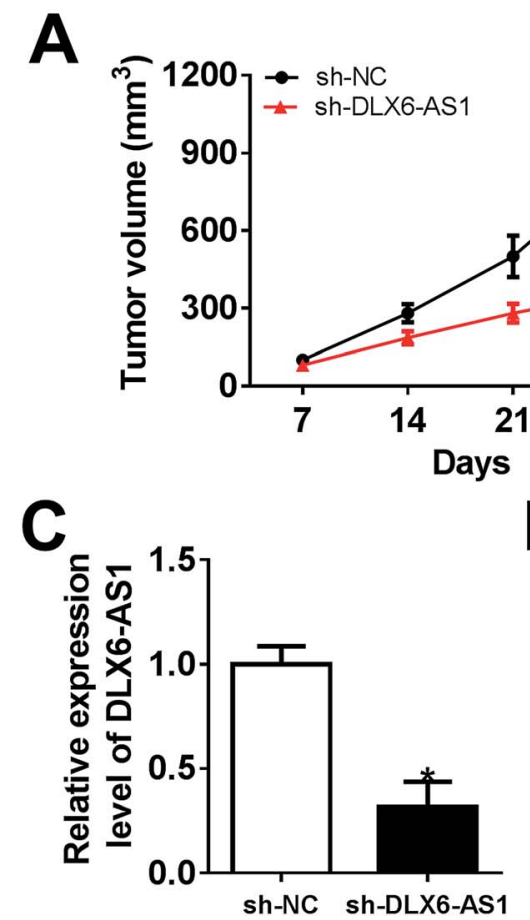

B
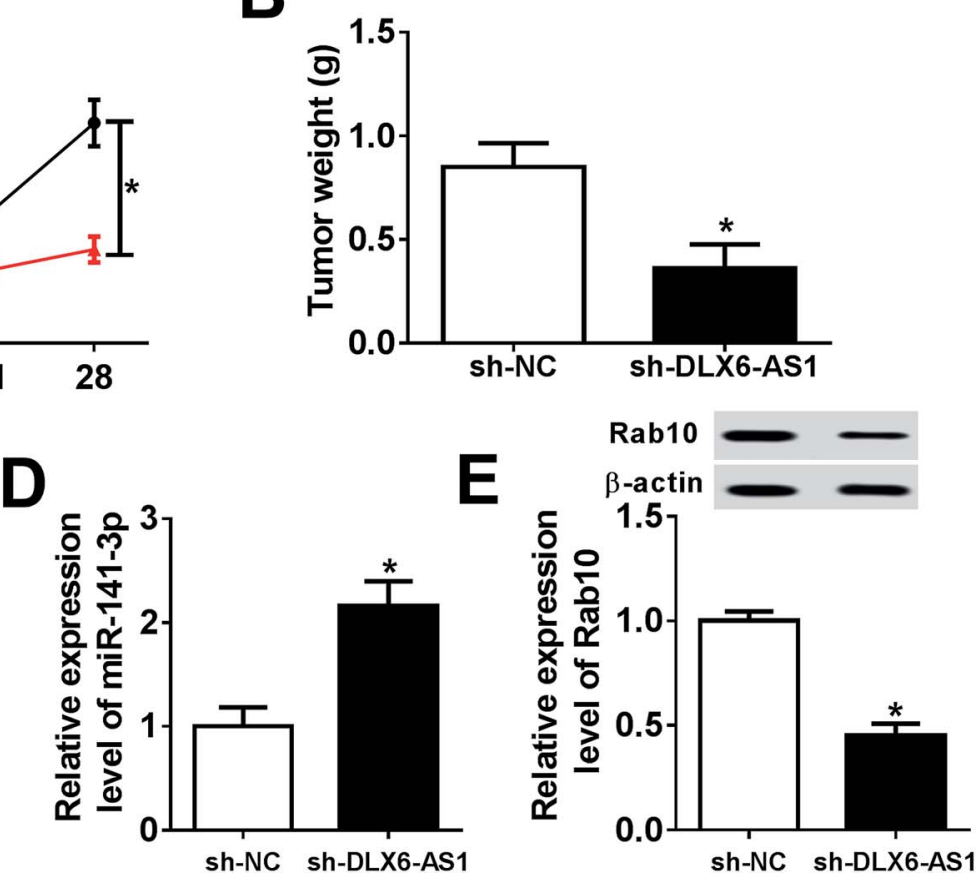

Fig. 8 Knockdown of DLX6-AS1 inhibited the tumor growth of MG-63 cells in vivo. MG-63 cells stably and lowly expressed DLX6-AS1 by the lentiviral infection of sh-DLX6-AS1 prior to injection into nude mice. (A) The volumes were calculated every 7 days after inoculation and the growth curve was drawn. (B) Tumor weight was recorded on day 28. (C-E) Expression of DLX6-AS1, miR-141-3p and Rab10 was confirmed in the xenograft tumors using RT-qPCR. $* P<0.05$. 
61 IncRNAs were expressed differently in MG-63 cells-derived mammospheres and DLX6-AS1 expression was more than 10fold higher than that in wild type MG63 and U2OS cells. This high expression predicted a poor prognosis. Functionally, the upregulation of DLX6-AS1 enhances OS stemness in MG-63 and U2OS cells through competitively interacting with miR-129-5p to DLK1, resulting in the activation of the Wnt signaling pathway. Another study put forward a significant correlation between higher DLX6-AS1 expression and advanced TNM stage, high tumor grade and distant metastasis in patients with OS. ${ }^{18}$ In that study, the knockdown of DLX6-AS1 could suppress OS cell proliferation, migration and invasion, and induce apoptosis in Saos-2 and U2OS cells through targeting the miR-641/HOXA9 axis. Here, we observed upregulated DLX6-AS1 in femoral OS tissues and cells as compared to in adjacent normal tissues and normal human osteoblast NHost cells, which was consistent with previous studies. ${ }^{\mathbf{1 8 , 1 9}}$ Moreover, the knockdown of DLX6AS1 not only inhibited OS cell proliferation, migration and invasion in MG-63 and U2OS cells, but also restricted the tumor growth of MG-63 cells in vivo through downregulating Rab10 by acting as a ceRNA for miR-141-3p. Taken together, it was concluded that DLX6-AS1 suppresses OS tumor growth in vivo as confirmed by xenograft experiments with DLX6-AS1-silenced Saos-2 and U2OS cells ${ }^{\mathbf{1 8 , 1 9}}$ as well as MG-63 cells in this study. Our data provide a novel DLX6-AS1/miR-141-3p/Rab10 pathway for regulating OS progression.

Rab10 belongs to the RAS superfamily of small GTPases ${ }^{28}$ and participates in glucose transporter type 4 (GLUT4) translocation, basement membrane secretion and endoplasmic reticulum (ER) formation and maintenance. ${ }^{29-31}$ To date, Rab10 was noticed to be activated under conditions of nutrient deprivation or mTOR inhibition. ${ }^{32}$ Acting as an oncogene, Rab10 was highly expressed in some malignant diseases, such as hepatocellular carcinoma, esophageal squamous cell carcinoma and acute myeloid leukemia. ${ }^{33-35}$ Several miRNAs were revealed to target Rab10, including miR-203, miR-378a-3p and miR-329. ${ }^{27,34,35}$ In OS, Rab10 was firstly reported to be targeted by miR-329 and the low expression of Rab10 inhibited OS cell proliferation and migration in MG-63 cells. ${ }^{27}$ Here, we found that Rab10 stood out as a promising candidate for miR-141-3p after software prediction, and the luciferase reporter assay further verified this target relationship in MG-63 and U2OS cells. Additionally, to the best of our knowledge, this is the first report on the upregulation of Rab10 in OS tissues and cells. Functionally, Rab10 overexpression could facilitate os cell proliferation, migration and invasion in MG-63 and U2OS cells, which supports the proposal of the Rab10 oncogenic role in OS by Jiang et $a .^{27}$

In this study, we provide the first evidence of the target relationship between miR-141-3p and either DLX6-AS1 or Rab10. Moreover, our results suggest a novel miR-141-3p/Rab10 axis underlying DLX6-AS1 in regulating OS cell progression both in vitro and in vivo. However, the role of the DLX6-AS1/miR-1413p/Rab10 pathway in other cell processes such as apoptosis ${ }^{18}$ remains to be further uncovered in OS, as well as its involvement in signaling pathways such as STAT3, Wnt/ $\beta$-catenin and Notch. ${ }^{15,16,36}$ Furthermore, a better understanding of the DLX6-
AS1 effect on drug resistance ${ }^{13,37}$ in OS should be urgently addressed for it to be an attractive therapeutic target for patients with OS.

In conclusion, the findings of this study support the tumorsuppressive role of DLX6-AS1 in OS. The results demonstrate that the knockdown of DLX6-AS1 caused the inhibition of proliferation, migration and invasion in MG-63 and U2OS cells through targeting the miR-141-3p/Rab10 axis. Moreover, the tumor growth of MG-63 cells in vivo was also inhibited with DLX6-AS1 downregulation. Our work suggests novel insights into the development and treatment of OS.

\section{Conflicts of interest}

There are no conflicts of interest to declare.

\section{References}

1 G. Ottaviani and N. Jaffe, Cancer Treat. Res., 2009, 152, 3-13, DOI: 10.1007/978-1-4419-0284-9_1.

2 A. D. Slade, C. L. Warneke, D. P. Hughes, P. A. Lally, K. P. Lally, A. A. Hayes-Jordan and M. T. Austin, J. Pediatr. Surg., 2015, 50, 157-160, DOI: 10.1016/ j.jpedsurg.2014.10.038.

3 A. B. Mohseny, K. Szuhai, S. Romeo, E. P. Buddingh, I. Briaire-de Bruijn, D. de Jong, M. van Pel, A. M. CletonJansen and P. C. Hogendoorn, J. Pathol., 2009, 219, 294305, DOI: 10.1002/path.2603.

4 N. Jaffe, A. Puri and H. Gelderblom, Sarcoma, 2013, 2013, 203531, DOI: 10.1155/2013/203531.

5 M. S. Isakoff, S. S. Bielack, P. Meltzer and R. Gorlick, J. Clin. Oncol., 2015, 33, 3029-3035, DOI: 10.1200/JCO.2014.59.4895.

6 D. J. Harrison, D. S. Geller, J. D. Gill, V. O. Lewis and R. Gorlick, Expert Rev. Anticancer Ther., 2018, 18, 39-50, DOI: 10.1080/14737140.2018.1413939.

7 M. M. Hagleitner, E. S. de Bont and D. M. Te Loo, Sarcoma, 2012, 2012, 636405, DOI: 10.1155/2012/636405.

8 J. Sana, P. Faltejskova, M. Svoboda and O. Slaby, J. Transl. Med., 2012, 10, 103, DOI: 10.1186/1479-5876-10-103.

9 E. P. Consortium, Nature, 2012, 489, 57-74, DOI: 10.1038/ nature11247.

10 M. A. Smolle and M. Pichler, Noncoding RNAs, 2018, 4, DOI: 10.3390/ncrna4010007.

11 Z. Li, P. Dou, T. Liu and S. He, Cell. Physiol. Biochem., 2017, 42, 1407-1419, DOI: 10.1159/000479205.

12 C. Wang, J. Jing and L. Cheng, Invest. New Drugs, 2018, 36, 1116-1132, DOI: 10.1007/s10637-018-0624-7.

13 K. P. Zhu, C. L. Zhang, X. L. Ma, J. P. Hu, T. Cai and L. Zhang, Mol. Ther., 2019, 27, 518-530, DOI: 10.1016/ j.ymthe.2019.01.001.

14 V. B. Sampson, S. Yoo, A. Kumar, N. S. Vetter and E. A. Kolb, Front. Pediatr., 2015, 3, 69, DOI: 10.3389/fped.2015.00069.

15 D. M. Wu, Z. H. Zheng, Y. B. Zhang, S. H. Fan, Z. F. Zhang, Y. J. Wang, Y. L. Zheng and J. Lu, J. Exp. Clin. Cancer Res., 2019, 38, 237, DOI: 10.1186/s13046-019-1239-3.

16 J. Yang, Z. Ye, D. Mei, H. Gu and J. Zhang, Cancer Manage. Res., 2019, 11, 4209-4221, DOI: 10.2147/CMAR.S194453. 
17 Y. Huang, R. Ni, J. Wang and Y. Liu, Biomed. Pharmacother., 2019, 109, 1851-1859, DOI: 10.1016/j.biopha.2018.09.151.

18 N. Zhang, X. Meng, L. Mei, C. Zhao and W. Chen, J. Cell. Biochem., 2019, DOI: 10.1002/jcb.28426.

19 R. M. Zhang, T. Tang, H. M. Yu and X. D. Yao, Biochem. Biophys. Res. Commun., 2018, 507, 260-266, DOI: 10.1016/ j.bbrc.2018.11.019.

20 H. Xu, Q. Mei, C. Xiong and J. Zhao, Cell Biochem. Biophys., 2014, 69, 319-325, DOI: 10.1007/s12013-013-9801-7.

21 N. Wang, P. Li, W. Liu, N. Wang, Z. Lu, J. Feng, X. Zeng, J. Yang, Y. Wang and W. Zhao, Oncol. Rep., 2018, 39, 747754, DOI: 10.3892/or.2017.6150.

22 K. Liu, Y. Hou, Y. Liu and J. Zheng, J. Biomed. Sci., 2017, 24, 46, DOI: 10.1186/s12929-017-0353-9.

23 H. H. Al-Khalaf and A. Aboussekhra, J. Biol. Chem., 2014, 289, 31433-31447, DOI: 10.1074/jbc.M114.593004.

24 J. Wang, G. Wang, B. Li, C. Qiu and M. He, OncoTargets Ther., 2018, 11, 4461-4478, DOI: 10.2147/OTT.S171304.

25 J. Li, P. Li, W. Zhao, R. Yang, S. Chen, Y. Bai, S. Dun, X. Chen, Y. Du, Y. Wang, W. Zang, G. Zhao and G. Zhang, Cancer Cell Int., 2015, 15, 48, DOI: 10.1186/s12935-015-0201-5.

26 P. He, Z. Zhang, G. Huang, H. Wang, D. Xu, W. Liao and Y. Kang, Am. J. Transl. Res., 2016, 8, 1780-1788.

27 W. Jiang, J. Liu, T. Xu and X. Yu, FEBS Lett., 2016, 590, 29732981, DOI: 10.1002/1873-3468.12337.

28 O. V. Vieira, Small GTPases, 2018, 9, 349-351, DOI: 10.1080/ 21541248.2016.1235004.
29 H. Sano, G. R. Peck, A. N. Kettenbach, S. A. Gerber and G. E. Lienhard, J. Biol. Chem., 2011, 286, 16541-16545, DOI: $10.1074 /$ jbc.C111.228908.

30 D. W. Lerner, D. McCoy, A. J. Isabella, A. P. Mahowald, G. F. Gerlach, T. A. Chaudhry and S. Horne-Badovinac, Dev. Cell, 2013, 24, 159-168, DOI: 10.1016/ j.devcel.2012.12.005.

31 A. R. English and G. K. Voeltz, Nat. Cell Biol., 2013, 15, 169178, DOI: $10.1038 / \mathrm{ncb} 2647$.

32 Z. Li, R. J. Schulze, S. G. Weller, E. W. Krueger, M. B. Schott, X. Zhang, C. A. Casey, J. Liu, J. Stockli, D. E. James and M. A. McNiven, Sci. Adv., 2016, 2, e1601470, DOI: 10.1126/ sciadv.1601470.

33 W. Wang, W. D. Jia, B. Hu and Y. Y. Pan, Oncotarget, 2017, 8, 26434-26447, DOI: 10.18632/oncotarget.15507.

34 N. Ding, X. Sun, T. Wang, L. Huang, J. Wen and Y. Zhou, Int. J. Mol. Med., 2018, 42, 381-391, DOI: 10.3892/ ijmm.2018.3639.

35 H. Fan, Y. Li, C. Liu, Y. Liu, J. Bai and W. Li, Biochem. Biophys. Res. Commun., 2018, 507, 178-184, DOI: 10.1016/ j.bbrc.2018.11.002.

36 J. Zhao and H. R. Liu, Eur. Rev. Med. Pharmacol. Sci., 2019, 23, 3243-3252, DOI: 10.26355/eurrev_201904_17684.

37 Y. Wang, L. Zhang, X. Zheng, W. Zhong, X. Tian, B. Yin, K. Tian and W. Zhang, Cancer Lett., 2016, 382, 137-146, DOI: 10.1016/j.canlet.2016.08.024. 